\title{
TWO NEW SPECIES OF BULBOPHYLLUM (ORCHIDACEAE) FROM THAILAND
}

\author{
JaAp J. Vermeulen ${ }^{1,5}$, Santi Watthana $^{2}$ \& Henrik Æ. Pedersen ${ }^{3,4}$ \\ ${ }^{1} \mathrm{Jk}$.art and science, Lauwerbes 8, 2318 AT Leiden, The Netherlands. \\ ${ }^{2}$ School of Biology, Institute of Science, Suranaree University of Technology, 111 University Avenue, \\ Suranaree, Muang, Nakhon Ratchsima, 30000, Thailand \\ ${ }^{3}$ Natural History Museum of Denmark, University of Copenhagen, \\ Gothersgade 130, DK-1123 Copenhagen, Denmark \\ ${ }^{4}$ Herbarium, Science, Royal Botanic Gardens, Kew, Richmond, Surrey, \\ England TW9 3AE, United Kingdom \\ ${ }^{5}$ Author for correspondence: jk.artandscience@gmail.com
}

\begin{abstract}
Aвstract. Two new species of Bulbophyllum (Orchidaceae) are described: B. sphenoglossum (sect. Lemniscata) and B. trigonanthum (sect. Reptantia). Both species occur in Thailand.

Key words/Palabras clave: Bulbophyllum sect. Lemniscata, Bulbophyllum sect. Reptantia, orquídeas, orchids, Tailandia, Thailand
\end{abstract}

Introduction. During revision of Bulbophyllum for the Flora of Thailand, we found two undescribed species in the collection of spirit samples brought together by Gunnar Seidenfaden and stored in C. Both species are only known from rather fragmentary samples and the limited information with these samples. Nevertheless, the material is sufficient to establish their identity and describe their morphology in detail, even though the paragraphs on flower color and habitat preferences are somewhat stark and incomplete. The plant habits in figures 1 and 2 are drawn from assembled parts of the plants in the samples.

\section{TAXONOMIC TREATMENT}

Bulbophyllum sphenoglossum J.J.Verm., Watthana \& H.A.Pedersen, sp. nov. (Fig. 1).

Bulbophyllum wallichii auct. Seidenfaden (1973): 234; (1979): 189 (both for specimen GT 5488 only).

Not Bulbophyllum wallichii Rchb.f. (1861): 259.

TYPE: Thailand. Chiang Mai Prov.: Doi Pha Hom Pok, Seidenfaden \& Smitinand GT 9534 (holotype: C).

Diagnosis: A species of sect. Lemniscata Pfitz. (Pfitzer 1889 [1888-1889]: 179; see also Vermeulen et al. 2014:
31 , characterized by 2-leaved pseudobulbs and deciduous leaves around anthesis. Resembles $B$. wallichii Rchb.f. because the two share the following set of character states: lowermost floral bract (ovate-)triangular; median sepal (long-)acuminate, margins distinctly erosefimbriate; lateral sepals adnate along their lower margin; lip approximately glabrous adaxially, hirsute abaxially. Differs from $B$. wallichii by the lip, which is $3.4-5.5 \mathrm{~mm}$ long (vs. 2.2-2.7 mm), and which distally is only slightly convex on the adaxial side (vs. distinctly convex and with a callus).

Plant rather small, a clustered epiphyte, roots spreading. Rhizome 2.0-3.8 mm diam., sections between pseudobulbs $0.8-1.5 \mathrm{~cm}$ long. Pseudobulbs 2-leaved, depressed ovoid to depressed conical, apex often drawn-out, 1.5-3.3 × 1.6-2.8 cm. Leaves deciduous at anthesis, not seen. Inflorescences racemose, 8-28 cm long, 5-16-flowered; peduncle 6-22 cm long, not thickened, rachis $1.4-6.0 \mathrm{~cm}$ long, floral bracts (ovate-)triangular, 3.6-7.0 × 2.2-3.0 mm, acuminate; 3-veined. Pedicel and ovary 2.5-3.2 mm long. Dorsal sepal porrect, triangular, $6.4-8.0 \times 1.8-2.9 \mathrm{~mm}$, ratio length/width 3.2-3.6; (long-)acuminate, margins distinctly erose-fimbriate, adaxially hirsute distally, 3-veined. Lateral sepals similar but lower margins 

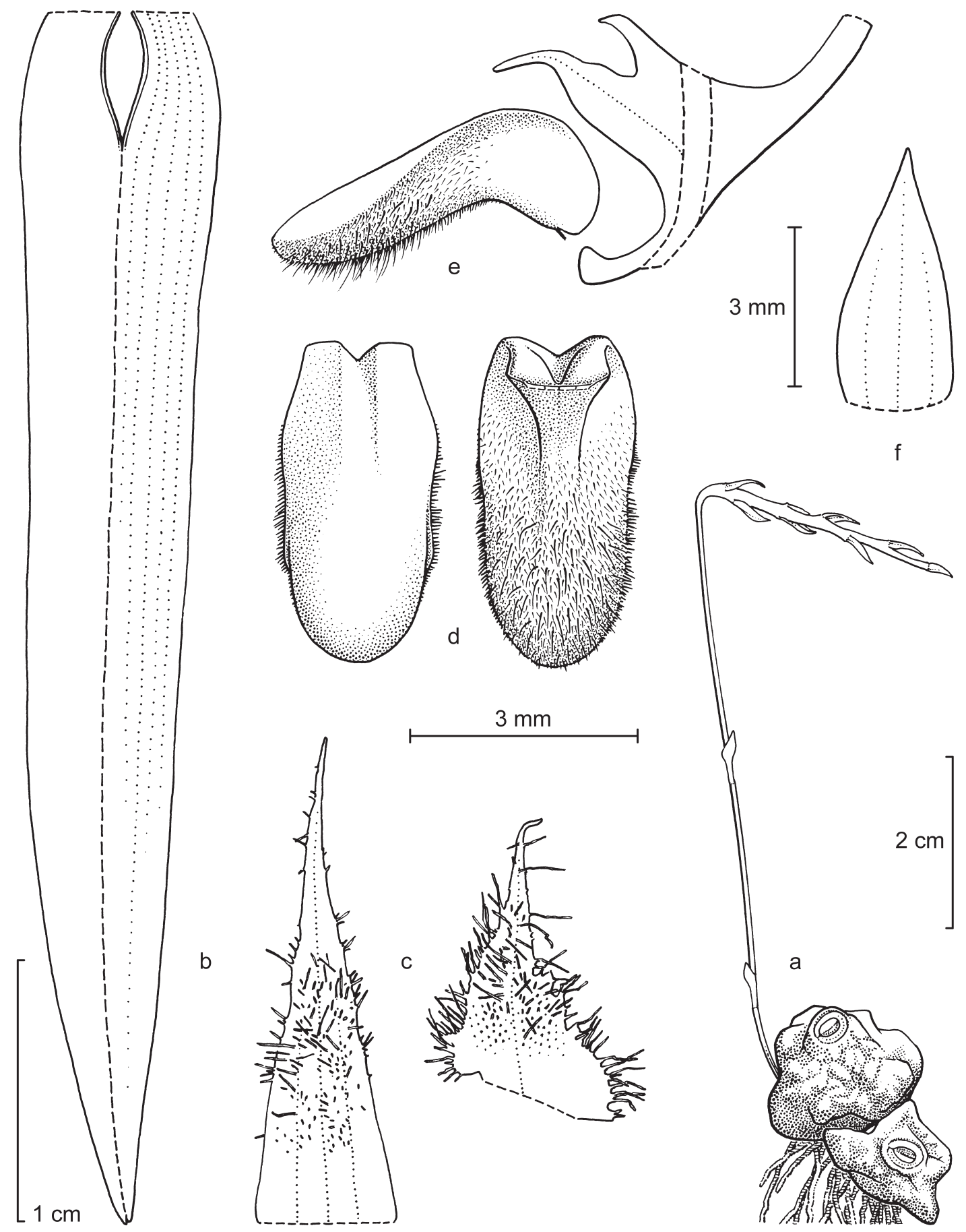

FIgURE 1. Bulbophyllum sphenoglossum J.J.Verm., Watthana \& H.A.Pedersen. a. Habit. b. Lateral sepals. c. From left to right: median sepal, petal. d. Lip, left: adaxial side, right: abaxial side. e. Column and lip, lateral view. f. Floral bract. Drawn from Seidenfaden \& Smitinand GT 9534, type (Drawn by C J.J. Vermeulen, from spirit material). 


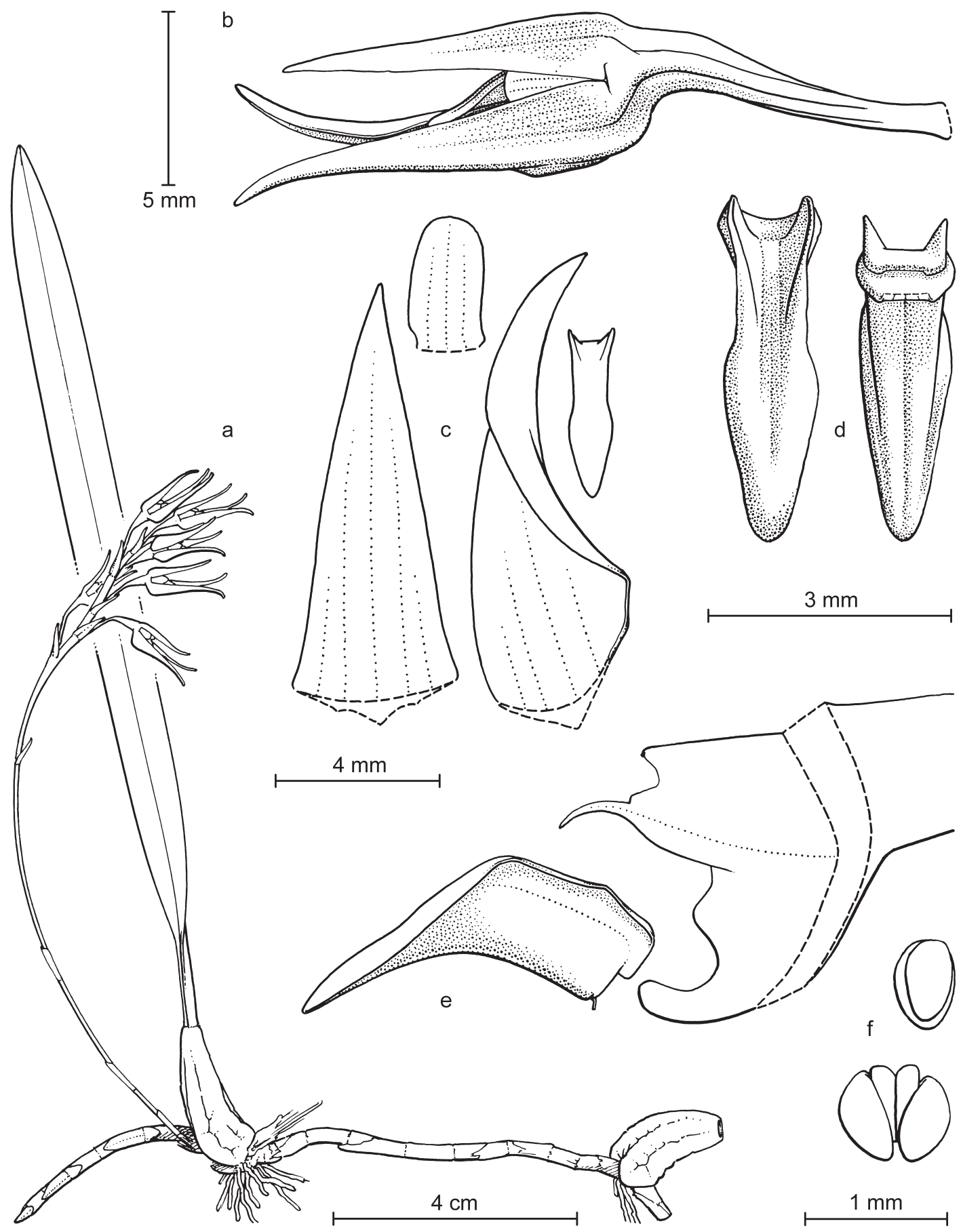

FIgURE 2. Bulbophyllum trigonanthum J.J.Verm., Watthana \& H.A.Pedersen. a. Habit. b. Flower. c. Flower analysis, from left to right: median sepal, petal, lateral sepal, lip. d. Lip left: adaxial side, right: abaxial side. e. Column and lip, lateral view. f. Pollinia, above: one pair, below: two pairs. Drawn from: a. Seidenfaden \& Smitinand GT 3077; b-f. Seidenfaden \& Smitinand GT 8927, type (Drawn by (C) J.J. Vermeulen, from spirit material). 
adnate except near base; oblique, ovate-oblong, 38-46 $\times 2.5-4.0 \mathrm{~mm}$, ratio length/width 12.0-15.2; acute, margins entire; glabrous, 5-veined. Petals recurved, obliquely triangular, $2.8-3.8 \times 1.9-2.0 \mathrm{~mm}$, ratio length/width 1.4-2.0; acuminate, margins fimbriate; adaxially papillose to hirsute distally, 1-veined. Lip recurved, ovate-oblong, 3.4-5.5 × 1.4-2.3 mm, ratio length/width 2.0-2.5 (without spreading); rounded, margins entire, shortly ciliate distally; adaxially slightly concave proximally, slightly convex distally, surface glabrous; abaxially shortly hirsute and with scattered long hairs distally. Column including stellidia 2.8-3.0 $\mathrm{mm}$ long; foot stipitate before widened apex. Stellidia downwards falcate, triangular, 1.5-1.9 mm long, acute, lower margin with a slight wing.

Colours: Unknown.

HabitAT AND ECOLOGY: Unknown; elevation 1800 m.

Material examined: Thailand. Chiang Mai Prov.: Doi Pha Hom Pok ('Doi Phaphompak', 'Doi Pahompok'), Seidenfaden \& Smitinand GT 9534 (C), GT 9542 (C). Phitsanulok Prov.: Phu Soi Dao ('Phu Mieng'), Seidenfaden \& Smitinand GT 5488 (C).

Etymology: The name is derived from Ancient Greek ' $\sigma \phi \eta \dot{\eta} v$ ', wedge, and ' $\gamma \lambda \omega \sigma \sigma \alpha$ ' tongue, referring to the distally thinning lip.

Note: One sample found in the lot identified as $B$. wallichii in Seidenfaden (1979), and two more samples collected after this date.

Bulbophyllum trigonanthum J.J.Verm., Watthana \& H.A.Pedersen, sp. nov. (Fig. 2)

Bulbophyllum luanii auct. Seidenfaden (1979): 109, fig. 69.

Not Bulbophyllum luanii Tixier in Guillaumin (1964): 396.

TYPE: Thailand. Loei Prov.: Phu Luang, Lon Tae, Seidenfaden \& Smitinand GT 8927 (holotype: C).

Diagnosis: A species of sect. Reptantia J.J.Verm. (in Pearce et al. 2001: 121; see also Vermeulen et al. 2014: 36), characterized by the racemose, synanthous inflorescences developing at the base of young shoots. Identified among species of this section (including
B. luanii Tixier from the same section) by the adaxially sharply keeled sepals, which give the buds and hardly opened flowers a triangular appearance when observed frontally.

Plant rather small, a long-creeping epiphyte, roots spreading. Rhizome 1.6-3.6 mm diam., sections between pseudobulbs $3.4-6.0 \mathrm{~cm}$ long. Pseudobulbs ovoid to lenticular, $1.1-2.3 \times 0.6-1.2 \mathrm{~cm}$. Leaves persistent, blade elliptic-oblong, $5.2-12.4 \times 0.5-1.1 \mathrm{~cm}$, ratio length/width $8.4-15.2$; obtuse; petiole $0.6-1.8$ $\mathrm{cm}$ long. Inflorescence (rather) lax-racemose, 6.6-19.3 cm long, 3-12-flowered; peduncle $5.5-13.5 \mathrm{~cm}$ long; rachis $1.1-7.5 \mathrm{~cm}$ long, floral bracts $4.0-8.0 \mathrm{~mm}$ long. Pedicel plus ovary $9.0-12.0 \mathrm{~mm}$ long. Dorsal sepal porrect, triangular, $7.6-10.7 \times 3.0-4.1 \mathrm{~mm}$, ratio length/width 2.5-2.9; acute, margins entire; glabrous, 3-5-veined, midvein abaxially prominent as a sharp keel. Lateral sepals similar but distally recurved, 9.0-11.6 × 3.0-4.1 mm, ratio length/width 2.3-2.5; 4-5-veined. Petals porrect, oblong, 2.9-3.5 × 1.6-1.9 $\mathrm{mm}$, ratio length/width 1.8-2.0; subtruncate to rounded, margins entire; glabrous, 3-veined. Lip recurved, (obovate-)oblong, 2.6-4.3 × 1.0-1.4 mm, ratio length/ width 2.6-2.8 (without spreading); obtuse to subacute, margins entire; glabrous; adaxially concave in the basal half, with two high ridges close to the edge, apical half slightly convex. Column including stelidia 2.5-3.5 mm long. Stelidia triangular with (short-)subulate apex, $1.0-1.3 \mathrm{~mm}$ long, acute; upper margin proximally with small tooth or erose, lower with slight, rounded to subacute wing proximally.

CoLours: Tepals yellow, stained red and with red veins; labellum yellow, stained red.

Habitat and ecology: Unknown. Elevation 1200$1300 \mathrm{~m}$.

Material examined: Thailand. Loei Prov:: Phu Kradueng ('Phu Krading'), Seidenfaden \& Smitinand GT 3061 (C), GT 3077 (C), GT 7943 (C); Phu Luang, Lon Tae, Seidenfaden \& Smitinand GT 8927 (C).

Eтymology: The name is derived from Ancient Greek ' $\chi \varrho i ́ \gamma \omega v o \varsigma$ ', triangular, and 'óv $\theta 0 \varsigma$ ', flower.

Note: Misidentified in Seidenfaden (1.c.) as B. luanii Tixier; the differences between the two species are given in the diagnosis. 


\section{LITERATURE CITED}

Guillaumin, A. (1964). Plantes Nouvelles, rares ou critiques des serres du museum (Notules sur quelques orchidées d'Indochine). Bulletin du Muséum National d'Histoire Naturelle. Paris, s.2, 36, 396-399.

Pearce, N. R., Cribb, P. J. \& Renz, J. (2001). Notes relating to the Flora of Bhutan 44. Taxonomic notes, new taxa, and additions to the Orchidaceae of Bhutan and Sikkim (India). Edinburgh Journal of Botany, 58, 99-122.

Pfitzer, E. H. H. (1888 - 1889). Orchidaceae. In: A. Engler \& K. Prantl, Die Natürlichen Pflanzenfamilien, 2(6), 52-220.

Reichenbach, H. G. (1861). Orchides. In: C. Mueller, Synopsis plantarum phanerogamicarum novarum omnium. Walpers Annales Botanices Systematicae, 6, 167-933.

Seidenfaden, G. (1973). Notes on Cirrhopetalum Lindl. Dansk Botanisk Arkiv, 29(1), 5-260.

Seidenfaden G. (1979). Orchid genera in Thailand 8: Bulbophyllum. Dansk Botanisk Arkiv, 33(3), 1-228.

Vermeulen, J. J., Fischer, G., Camargo Smidt, E. de, Stern, W. L., Pridgeon, A. M., Veitch, C., Sieder, A., Vugt, R. van \& Gravendeel, B. (2014). Bulbophyllum. In: A. M. Pridgeon, P. J. Cribb, M. W. Chase \& F. N. Ramussen (Eds.), Genera Orchidacearum volume 6, Epidendroideae pt. 3 (pp. 4-51). UK: Oxford University Press. 
LANKESTERIANA 